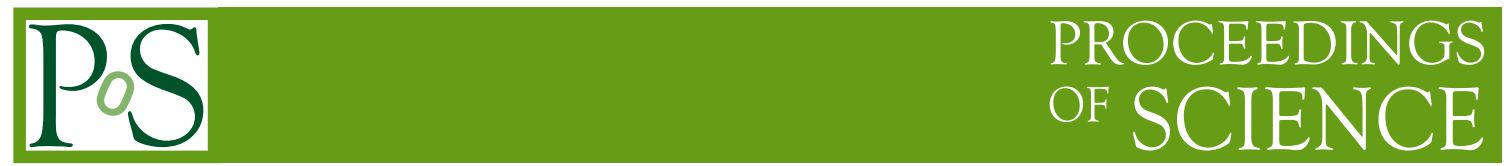

\title{
Dark Matter Candidates: Status and Perspectives
}

\author{
Tim M.P. Tait* \\ Department of Physics and Astronomy \\ University of California, Irvine \\ Irvine, CA 92697 \\ E-mail: ttait@uci.edu
}

\begin{abstract}
This review summarizes the talk on Dark Matter Candidates: Status and Perspectives during the plenary sessions of ICRC 15 in The Hague, Netherlands. It includes discussions of theoreticallymotivated candidates which could play the role of dark matter, as well as some highlights of the current status of direct, indirect, collider, and astronomical searches which aim to shed light on its properties and identify.
\end{abstract}

The 34th International Cosmic Ray Conference

30 July- 6 August, 2015

The Hague, The Netherlands

${ }^{*}$ Speaker. 


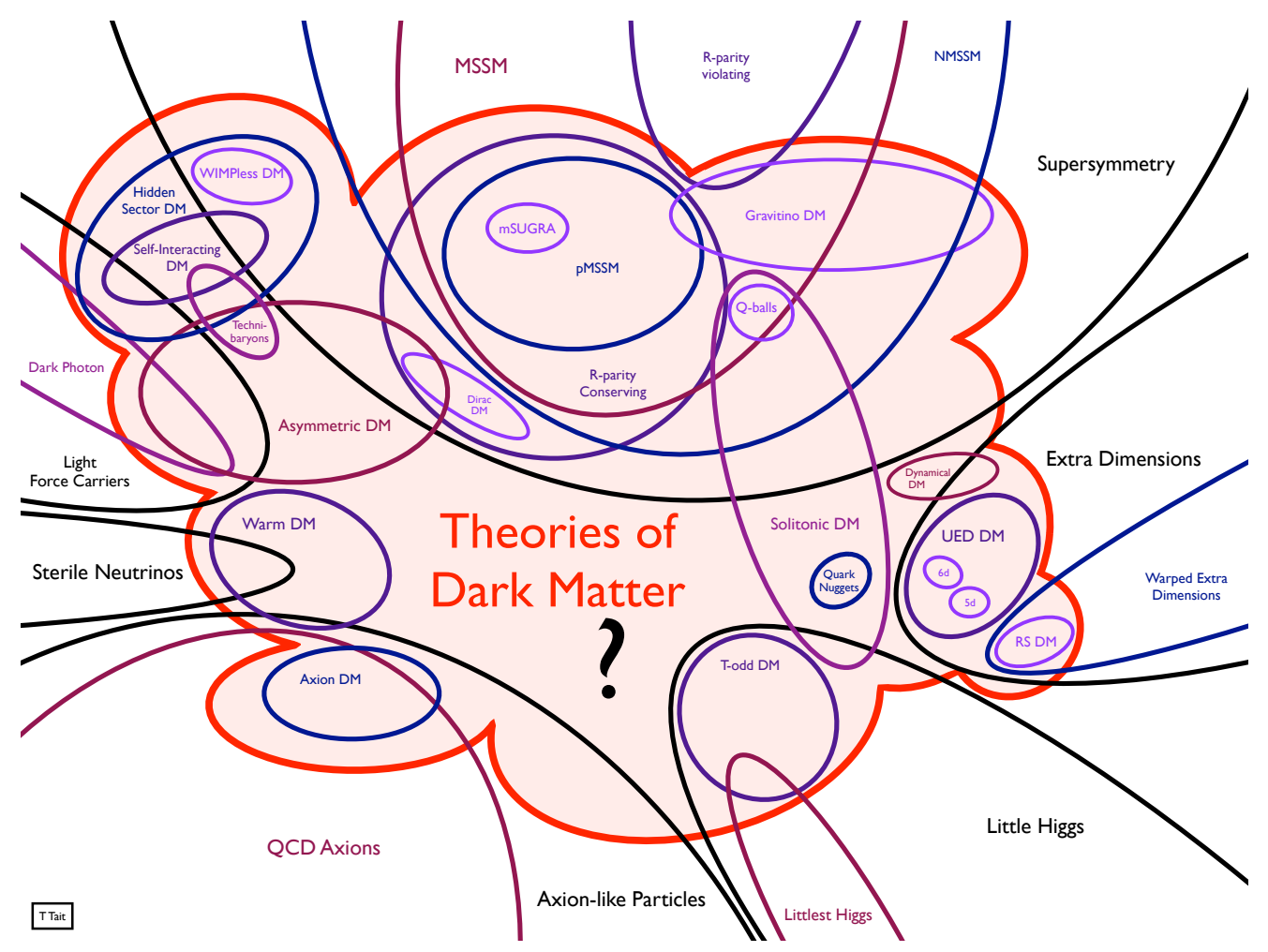

Figure 1: (Incomplete) Venn diagrams of theories of dark matter.

\section{Introduction}

The evidence for dark matter is overwhelming [1], and points to the need for what is most likely a new quantum field which must supplement the Standard Model (SM) of particle physics. The identification of this field is thus of paramount importance in order to extend the Standard Model. Seeing how dark matter fits together with the Standard Model structure is a likely to provide key insights into fundamental physics and may reveal new principles of Nature. A wide variety of experimental searches aimed at uncovering clues are underway. In this talk I provide an over-view of theoretical ideas for what could constitute the dark matter (Sec. 2) and discuss the current status of experimental searches (Sec. 3). I apologize in advance that because each of these areas are wide fields in themselves, my discussion will by necessity be somewhat personalized and incomplete. I must further apologize that references are largely to reviews or other talks at the conference, and are intended more as a starting point for an interested reader to learn more rather than a fair historical representation of the literature.

\section{Candidates}

There are a wide variety of the theoretical ideas as to what might constitute the dark matter (see Fig. 1). In terms of its particle physics properties, a viable dark matter candidate must satisfy 
the relatively modest requirements that it be:

- dark, not charged under either the SM's electromagnetic U(1) $)_{\mathrm{EM}}$ or strong nuclear SU(3)C interactions;

- cold, meaning that it was sufficiently non-relativistic at the time of structure formation; and

- stable, with a lifetime such that a significant fraction of it persists in the Universe today.

Nothing in the Standard Model itself possesses these properties without invoking additional ingredients, and a dizzying array of possibilities could represent the truth, or contain elements of truth. There are far too many to go through in detail, and so I limit my discussion to brief mention of sterile neutrinos, axions, and weakly-interacting massive particles (WIMPs).

\subsection{Sterile Neutrinos}

Dark matter may be intimately linked to another manifestation of physics beyond the Standard Model: the fact that neutrinos are observed to have masses, leading to them mixing when propagating over long distances. The simplest extension of the SM engineering masses for the neutrinos is to add additional Weyl fermions which are singlets under the SM gauge symmetries (and hence sterile). Such particles are allowed by symmetries to have Majorana masses and to interact with the combination of the Standard Model Higgs doublet together with the ordinary (active) neutrinos. After the Higgs acquires its vacuum expectation value and the fields are rotated to the mass basis, the theory describes six Majorana fermions which are mixtures of the original active and sterile fermions. Measurements of neutrino oscillations indicate that three of these states must be overwhelmingly composed of the active components, implying that the additional fermions are dominantly sterile.

These heavier states can decay into the lightest states (typically plus a photon) through the electroweak interaction. The rate of this decay is proportional to the small quantity of active neutrino that state contains, and for very small mixing a lifetime on the order of the age of the Universe would be viable with such a state playing the role of dark matter. For such a particle to be cold enough to be consistent with our understanding of structure formation requires that its mass be $m \gtrsim 10 \mathrm{keV}[2]$.

\subsection{Axions}

The axion is a hypothetical particle which arises from the Peccei-Quinn solution to the strong $\mathrm{CP}$ problem $[3,4,5]$. The problem boils down to the fact that the strong nuclear force inherently contains a parameter $\bar{\theta}$ consistent with all known symmetries which violates the discrete CP symmetry, and would induce an electric dipole moment (EDM) for the neutron. Increasingly sensitive measurements have failed to observe such a neutron EDM, and currently require $\bar{\theta} \lesssim 10^{-9}$. Such a tiny value seems profoundly unnatural, and begs for a dynamical explanation, which the axion provides by transforming it into a dynamical quantity which then relaxes to zero.

The fluctuations of this dynamical field appear as a pseudo-Goldstone boson whose interactions are characterized by $1 / f_{a}$ and mass is roughly $m_{a} \sim f_{\pi} / f_{a} m_{\pi}$. Observations require 
$f_{a} \gtrsim 10^{9} \mathrm{GeV}$ [2], indicating that the axion must be extremely light and extremely weakly coupled. The axion is also inherently unstable, but its tiny mass and coupling indicate that it can easily have a lifetime long enough for it to successfully play the role of dark matter.

\subsection{Weakly Interacting Massive Particles}

Particles with roughly electroweak scale masses and couplings occur in many popular extensions of the Standard Model, including supersymmetric versions [6, 7, 8], models with Universal Extra Dimensions [9, 10, 11, 12, 13], and realistic little Higgs theories [14, 15]. Even on top of their purely theoretical motivation, they make for an attractive dark matter candidate because their abundance in the early Universe can be understood from their freezing out from equilibrium. Since their density is determined by when they fall out of equilibrium with the SM plasma, the observed density of dark matter implies an annihilation rate of (assuming that their average cross section $\langle\sigma v\rangle$ is $s$-wave, or velocity-independent) of $3 \times 10^{-26} \mathrm{~cm}^{3} / \mathrm{s}$ [16]. Given their large masses and relatively strong coupling to the SM, it is generically necessary to impose a (perhaps very weakly broken) symmetry such that they are stable or long-lived enough to be dark matter.

\section{Probes}

Each of these visions for dark matter imply that it has some kind of interaction with ordinary matter. Thus, each leads to strategies for how it can be observed and identified. In this section, I begin with a quick review of the particle physics strategies for detecting dark matter, followed by a discussion of how they fit together, and close with some comments about astronomical probes.

\subsection{Direct Detection}

Direct searches for dark matter seek to observe the presence of the ambient dark matter in the solar system through its interaction with detectors on the Earth. As a result, such searches are sensitive to the precise density of dark matter in the neighborhood of the solar system, as well as to the distribution of its velocity.

One class of experiments builds very sensitive detectors aimed at seeing the dark matter colliding with a SM target (typically the nucleus of a heavy atom [17, 18], though scattering with electronic structure is also being investigated [19, 20,21]). Dark matter appears as an otherwise undetected particle coming in, scattering with the detector and producing a signal, and then exiting again without being detected. For dark matter with masses in the range of $\sim 10-100 \mathrm{GeV}$, remarkable advances in sensitivity spanning several orders of magnitude (see Fig. 2) have been achieved in a short range of time, and have effectively covered scenarios in which the dark matter interacts by exchange of a $Z$ boson, and are now closing in on the possibility of exchange of the newly discovered Higgs boson as well. Ultimately, such detectors will become sensitive to low energy neutrinos [22], which will slow down future increases in sensitivity. Another active target region is masses below $\sim 10 \mathrm{GeV}$, which require sensitivity to much smaller momentum transfer.

Ambient axons can also be detected, by using the fact that they typically couple to electric and magnetic fields, $\vec{E} \cdot \vec{B}$ with a coupling characterized by $1 / f_{a}$ (but with some dependence on the UV details of the axion model). In an experiment with a background magnetic field, an incoming axion can be converted by this interaction into a photon, whose presence can then be magnified and 


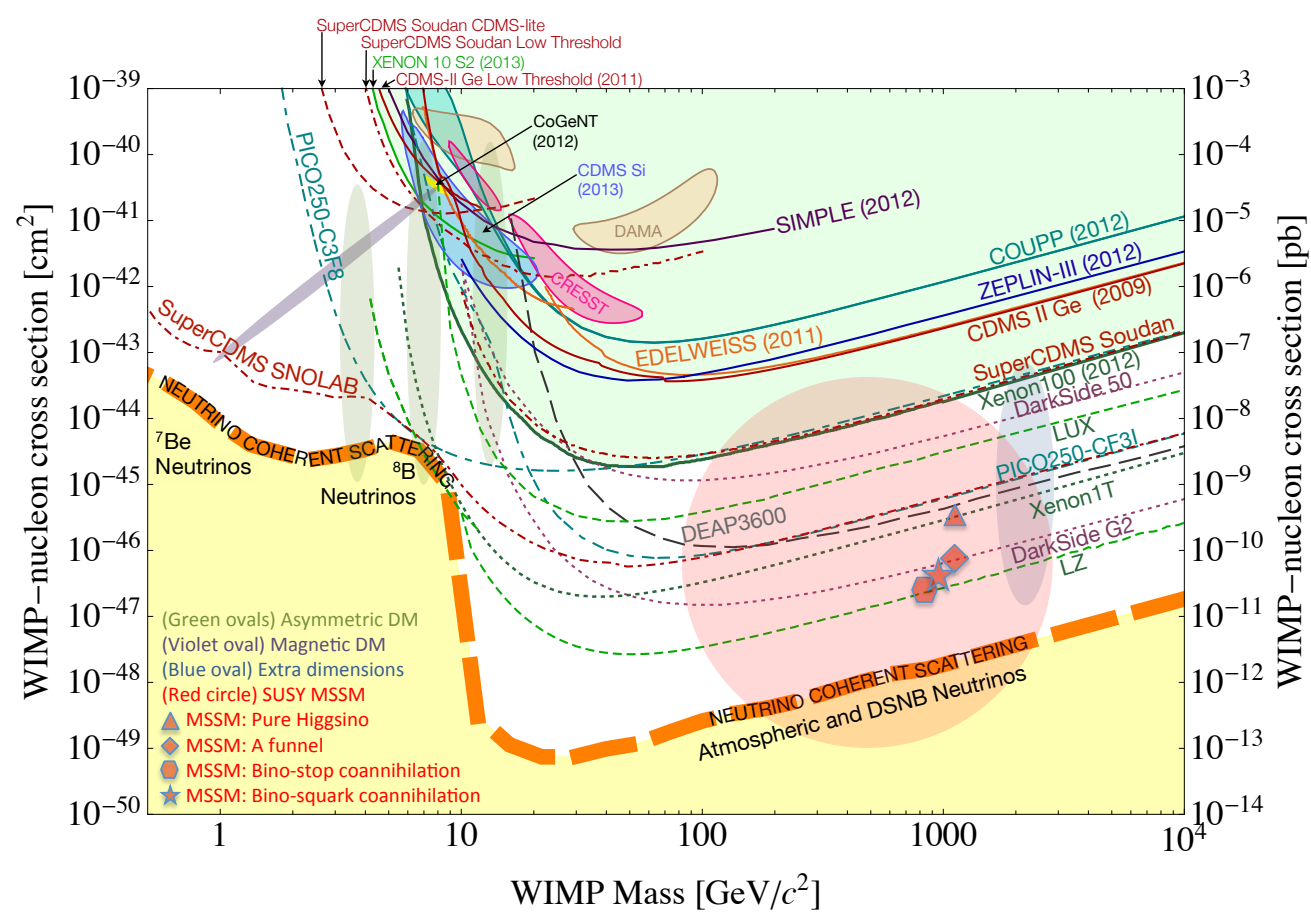

Figure 2: Constraints (and future projections) on the cross section for dark matter to scatter with a nucleon as a function of the dark matter mass. From Ref. [17].

detected [2]. Already, interesting regions of parameter space have been ruled out by these searches, and new ideas $[23,24]$ hold the promise to push into previously unexplored regions of parameter space.

\subsection{Collider Searches}

High energy colliders such as the LHC can hope to produce the dark matter directly. Since it is expected to interact much too weakly to leave a trace in the detectors which examine the collisions, its existence must be inferred from an imbalance in the net momentum of the observed products of the reaction. Since colliders do not rely on the dark matter being already present in the initial state, a positive signal would not establish the production of dark matter (as opposed to some other weakly interacting, but unstable state). Nonetheless, they have exquisite control over the initial state and painstakingly developed control over background processes. A positive signal would be highly suggestive and would help sharpen the direction of future direct and indirect searches.

There are many different searches by the ATLAS and CMS experiments which are relevant for theories of dark matter, including e.g. searches for supersymmetric particles [25, 26] and generic searches for anomalously large production of events with missing transverse momentum $[27,28]$. In order to connect such searches to the properties of dark matter, a theoretical construct is required. A variety are employed, including the phenomenological Minimal Supersymmetric Standard Model (pMSSM) [8], simplified models describing the dark matter and a particle mediating its interactions [29, 30], and effective field theories (EFTs) which are the universal 

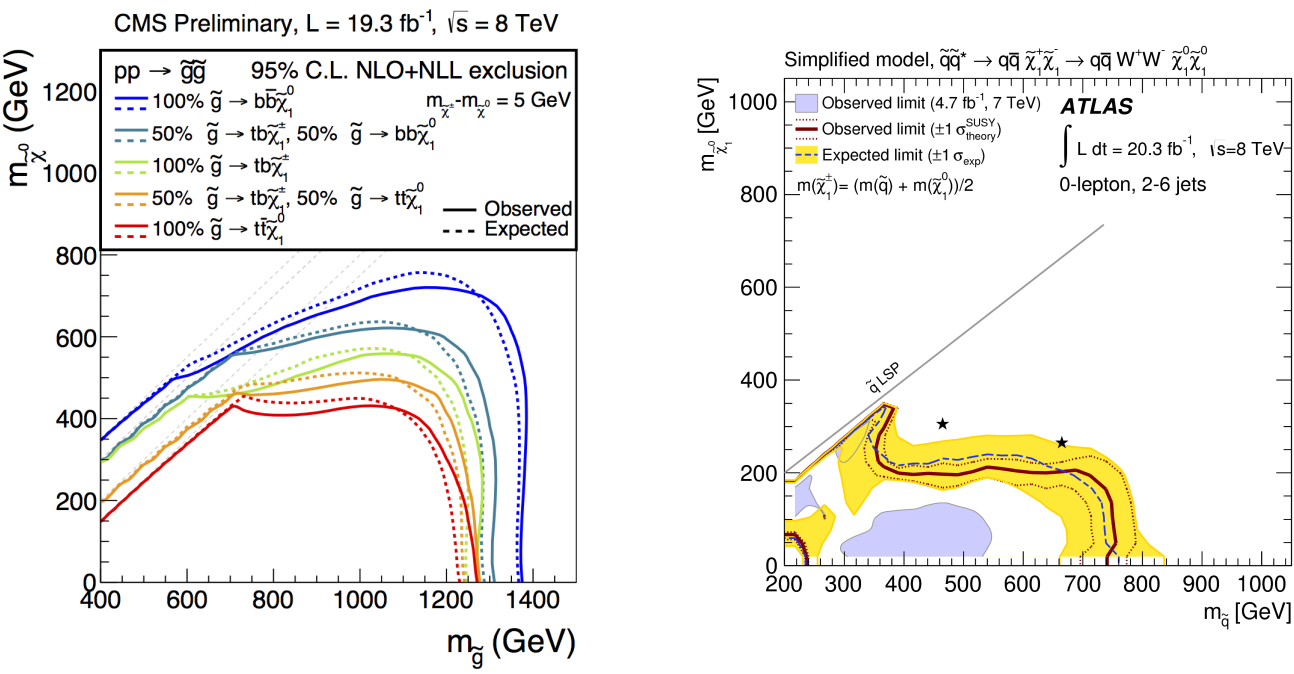

Figure 3: Constraints on parameter space of supersymmetric theories (as indicated) derived from searches for the indicated channels involving missing momentum at the LHC $[25,26]$.

limit of all models when the mediator particles are heavy compared to the energies of interest $[31,32,33,34,35,36,37,38]$. As two (of many possible) examples of the impact of such a search, Fig. 3 shows regions of supersymmetric parameter space excluded by searches for missing momentum plus jets of hadronic particles.

\subsection{Indirect Detection}

Indirect detection aims to look at the rare annihilation of two dark matter particles which is expected in regions where the dark matter is over-dense, such as the in centers of galaxies. While many of the products of such annihilation will themselves decay on their way from the annihilation point to the Earth, photons, neutrinos, and anti-matter may arrive, and could be distinctive enough from astrophysical sources for one to reconstruct their origin as a signal of dark matter [39]. Searches for dark matter annihilations resulting in gamma rays [40, 41, 42], neutrinos [44, 43], positrons [45, 46], and anti-protons [47] are all well underway. While charged particles are subject to the galactic magnetic fields, $\mathrm{GeV}-\mathrm{TeV}$ photons and neutrinos are expected to propagate on galactic scales with relatively little directional or energy loss. As a result, their distribution in the sky allows the direction of their origin to be reconstructed.

Features appear in these searches which are not well-described by modeling of astrophysical fore- and back-grounds. The positron excess above $10 \mathrm{GeV}$ first observed by PAMELA [45] and subsequently confirmed by AMS-02 [46] remains unexplained, with a dark matter interpretation requiring a cross section which is somewhat shockingly large, though not outside of the realm of theoretical engineering [48, 49]. More recently, analysis of gamma rays from the direction of the galactic center region collected by the Fermi LAT show an excess at $\sim \mathrm{GeV}$ energies [50, 51, 52, 53, 54], which is also observed in analysis by the Fermi collaboration itself [55, 56, 57]. In Fig. 4 is the spectrum of the excess for four different models of the astrophysical back- and fore-grounds. These spectra are roughly consistent with dark matter annihilation into hadronic final states when 

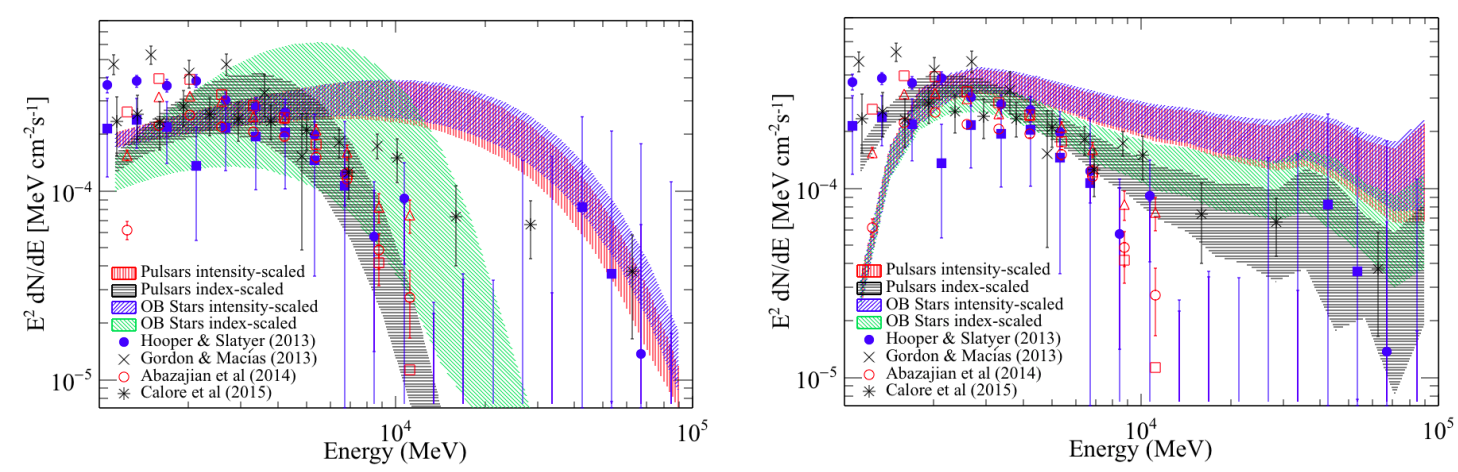

Figure 4: Spectrum of the excess of gamma rays from the galactic center [57] for four different models of the back- and fore-grounds. The spectra in the right plot are fixed to the functional form of a power law with exponential cut-off, while those on the right are fit in energy bins.

the dark mass is between about 30 and $120 \mathrm{GeV}$ [58], or even higher if the annihilation is into mediators which themselves decay into SM particles [59, 60, 61]. While tantalizing, it remains unclear whether this excess is in fact the result of dark matter annihilation, as opposed to some more prosaic explanation.

\subsection{Complementarity of Particle Probes}

A common theme among direct, indirect, and collider searches for dark matter is the fact that all three ultimately are testing the strength and nature of dark matter's interactions with the Standard Model. Thus, in some sense they can be compared as far as their particular strengths and weaknesses, leading to an understanding of how they complement one another [62,63]. In Fig. 5 we show the "current" reach (as of 2013) and future projections of all three types of searches translated into the annihilation cross section (normalized by the target cross section for a thermal relic saturating observations). The translation is performed in the limit in which the mediating particles are heavy (compared to the energy of any of the experiments) such that the interaction is described by an effective field theory of the form indicated on the figure. This exercise reveals broad trends, such as the fact that collider limits tend to be stronger on lighter dark matter particles, whereas indirect detection works best at larger masses and direct detection works best when the interaction allows for spin-independent scattering with heavy nuclei. Similar features are observed in more complete theories such as the pMSSM [64].

\subsection{Astronomical Probes}

Astronomy offers a unique window to properties of the dark matter which are difficult to otherwise access. For example, the distribution of dark matter in galaxy cluster mergers was long ago recognized to provide an upper limit on the cross section for dark matter to scatter elastically with itself [65]. More recently, self-interaction has been invoked to alleviate tensions (modulo systematic uncertainties from astrophysics and structure formation simulations) in the observations of galaxies $[66,67]$. The necessary cross sections are much larger than what would be expected for 

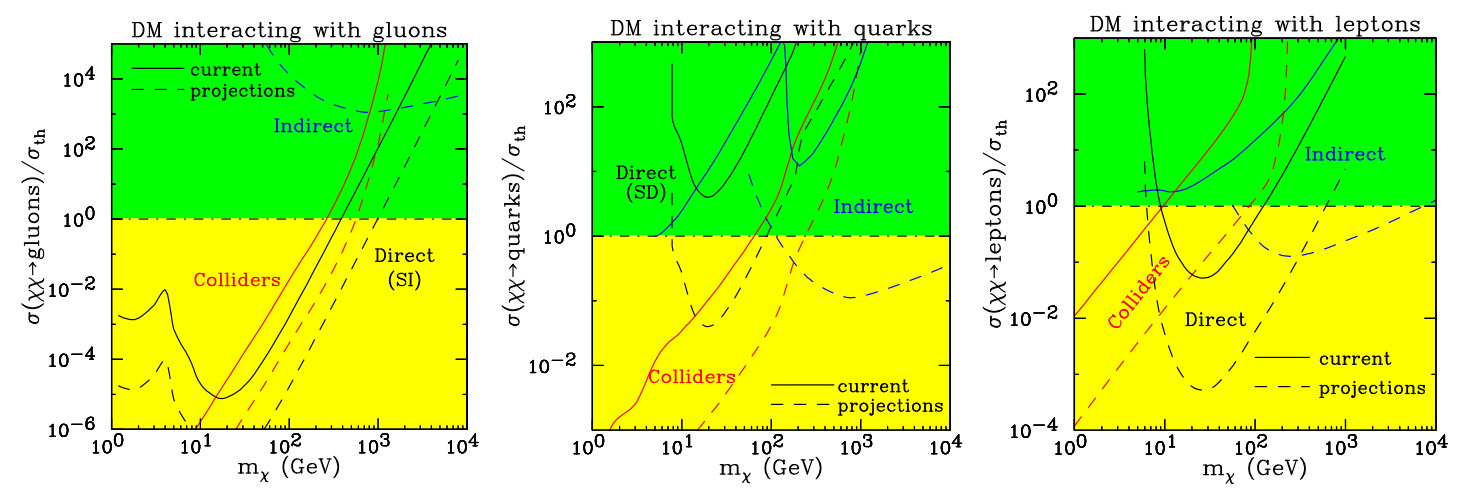

Figure 5: Current exclusions (as of 2013) and future projections of the reach of different classes of experiments for three types of dark matter interactions, presented as a bound on the annihilation cross section divided by the cross section leading to saturations of the observed relic density of dark matter. From [62].

a generic WIMP, more in line with hadron-hadron scattering rates. Already, this suggests interesting directions leading to such a large scattering cross section to explore, such as the possibility that the dark matter is a composite bound state of a hidden sector non-Abelian gauge symmetry $[68,69,70,71]$.

\section{Outlook}

The identity of the dark matter stands among the most pressing questions confronting particle physics today. There is a bewildering plethora of theoretical ideas under discussion, which a wide array of experimental activity aims to confirm or rule out. In this data rich era, there is good hope that we will soon discover clues as to the nature of dark matter. Uncovering those clues and combining them into a cogent picture of dark matter will take collaboration between theorists and experimentalists. If I had to guess, it will be confusing, surprising, and undoubtably a lot of fun!

\section{Acknowledgements}

I am happy to thank the organizers of ICRC 2015 for an excellent, stimulating meeting. My research is supported in part by NSF grant PHY-1316792 and by a Chancellor's Fellowship from the University of California, Irvine.

\section{References}

[1] G. Bertone, D. Hooper and J. Silk, Phys. Rept. 405, 279 (2005) doi:10.1016/j.physrep.2004.08.031 [hep-ph/0404175].

[2] A. Kusenko and L. J. Rosenberg, arXiv:1310.8642 [hep-ph].

[3] R. D. Peccei and H. R. Quinn, Phys. Rev. Lett. 38, 1440 (1977). doi:10.1103/PhysRevLett.38.1440

[4] S. Weinberg, Phys. Rev. Lett. 40, 223 (1978). doi:10.1103/PhysRevLett.40.223 
[5] F. Wilczek, Phys. Rev. Lett. 40, 279 (1978). doi:10.1103/PhysRevLett.40.279

[6] G. Jungman, M. Kamionkowski and K. Griest, Phys. Rept. 267, 195 (1996) doi:10.1016/0370-1573(95)00058-5 [hep-ph/9506380].

[7] A. Arbey, M. Battaglia and F. Mahmoudi, Eur. Phys. J. C 72, 1847 (2012) doi:10.1140/epjc/s10052-011-1847-3 [arXiv:1110.3726 [hep-ph]].

[8] R. C. Cotta, J. S. Gainer, J. L. Hewett and T. G. Rizzo, New J. Phys. 11, 105026 (2009) doi:10.1088/1367-2630/11/10/105026 [arXiv:0903.4409 [hep-ph]].

[9] T. Appelquist, H. C. Cheng and B. A. Dobrescu, Phys. Rev. D 64, 035002 (2001) doi:10.1103/PhysRevD.64.035002 [hep-ph/0012100].

[10] G. Servant and T. M. P. Tait, Nucl. Phys. B 650, 391 (2003) doi:10.1016/S0550-3213(02)01012-X [hep-ph/0206071].

[11] H. C. Cheng, J. L. Feng and K. T. Matchev, Phys. Rev. Lett. 89, 211301 (2002) doi:10.1103/PhysRevLett.89.211301 [hep-ph/0207125].

[12] K. Kong and K. T. Matchev, JHEP 0601, 038 (2006) doi:10.1088/1126-6708/2006/01/038 [hep-ph/0509119].

[13] F. Burnell and G. D. Kribs, Phys. Rev. D 73, 015001 (2006) doi:10.1103/PhysRevD.73.015001 [hep-ph/0509118].

[14] H. C. Cheng and I. Low, JHEP 0309, 051 (2003) doi:10.1088/1126-6708/2003/09/051 [hep-ph/0308199].

[15] J. Hubisz and P. Meade, Phys. Rev. D 71, 035016 (2005) doi:10.1103/PhysRevD.71.035016 [hep-ph/0411264].

[16] For recent refinements and original references, see: G. Steigman, B. Dasgupta and J. F. Beacom, Phys. Rev. D 86, 023506 (2012) doi:10.1103/PhysRevD.86.023506 [arXiv:1204.3622 [hep-ph]].

[17] P. Cushman et al., arXiv:1310.8327 [hep-ex].

[18] For a review, see : Enactali Figueroa-Feliciano, these proceedings (2015).

[19] R. Essig, J. Mardon and T. Volansky, Phys. Rev. D 85, 076007 (2012) doi:10.1103/PhysRevD.85.076007 [arXiv:1108.5383 [hep-ph]].

[20] Y. Hochberg, Y. Zhao and K. M. Zurek, arXiv:1504.07237 [hep-ph].

[21] R. Essig, M. Fernandez-Serra, J. Mardon, A. Soto, T. Volansky and T. T. Yu, arXiv:1509.01598 [hep-ph].

[22] J. Billard, L. Strigari and E. Figueroa-Feliciano, Phys. Rev. D 89, no. 2, 023524 (2014) doi:10.1103/PhysRevD.89.023524 [arXiv:1307.5458 [hep-ph]].

[23] P. W. Graham and S. Rajendran, Phys. Rev. D 88, 035023 (2013) doi:10.1103/PhysRevD.88.035023 [arXiv:1306.6088 [hep-ph]].

[24] P. Sikivie, N. Sullivan and D. B. Tanner, Phys. Rev. Lett. 112, no. 13, 131301 (2014) doi:10.1103/PhysRevLett.112.131301 [arXiv:1310.8545 [hep-ph]].

[25] L. S. Ancu [ATLAS Collaboration], arXiv:1412.2784 [hep-ex].

[26] A. Gaz [CMS Collaboration], arXiv:1411.1886 [hep-ex]. 
[27] G. Aad et al. [ATLAS Collaboration], JHEP 1304, 075 (2013) doi:10.1007/JHEP04(2013)075 [arXiv:1210.4491 [hep-ex]].

[28] V. Khachatryan et al. [CMS Collaboration], Eur. Phys. J. C 75, no. 5, 235 (2015) doi:10.1140/epjc/s10052-015-3451-4 [arXiv:1408.3583 [hep-ex]].

[29] J. Abdallah et al., Phys. Dark Univ. 9-10, 8 (2015) doi:10.1016/j.dark.2015.08.001 [arXiv:1506.03116 [hep-ph]].

[30] D. Abercrombie et al., arXiv:1507.00966 [hep-ex].

[31] M. Beltran, D. Hooper, E. W. Kolb and Z. C. Krusberg, Phys. Rev. D 80, 043509 (2009) doi:10.1103/PhysRevD.80.043509 [arXiv:0808.3384 [hep-ph]].

[32] M. Beltran, D. Hooper, E. W. Kolb, Z. A. C. Krusberg and T. M. P. Tait, "Maverick dark matter at colliders," JHEP 1009, 037 (2010) [arXiv:1002.4137 [hep-ph]].

[33] J. Goodman, M. Ibe, A. Rajaraman, W. Shepherd, T. M. P. Tait and H. -B. Yu, "Constraints on Light Majorana dark Matter from Colliders," Phys. Lett. B 695, 185 (2011) [arXiv:1005.1286 [hep-ph]].

[34] Y. Bai, P. J. Fox and R. Harnik, "The Tevatron at the Frontier of Dark Matter Direct Detection," JHEP 1012, 048 (2010) [arXiv:1005.3797 [hep-ph]].

[35] J. Goodman, M. Ibe, A. Rajaraman, W. Shepherd, T. M. P. Tait and H. -B. Yu, "Constraints on Dark Matter from Colliders,” Phys. Rev. D 82, 116010 (2010) [arXiv:1008.1783 [hep-ph]].

[36] A. Rajaraman, W. Shepherd, T. M. P. Tait and A. M. Wijangco, "LHC Bounds on Interactions of Dark Matter," Phys. Rev. D 84, 095013 (2011) [arXiv:1108.1196 [hep-ph]].

[37] P. J. Fox, R. Harnik, J. Kopp and Y. Tsai, "Missing Energy Signatures of Dark Matter at the LHC," Phys. Rev. D 85, 056011 (2012) [arXiv:1109.4398 [hep-ph]].

[38] K. Cheung, P. Y. Tseng, Y. L. S. Tsai and T. C. Yuan, "Global Constraints on Effective Dark Matter Interactions: Relic Density, Direct Detection, Indirect Detection, and Collider," JCAP 1205, 001 (2012) [arXiv:1201.3402 [hep-ph]].

[39] For expanded discussion, see: Marco Cirelli, these proceedings (2015).

[40] M. Ackermann et al. [Fermi-LAT Collaboration], Phys. Rev. Lett. 115, no. 23, 231301 (2015) doi:10.1103/PhysRevLett.115.231301 [arXiv:1503.02641 [astro-ph.HE]].

[41] A. Abramowski et al. [HESS Collaboration], Phys. Rev. D 90, 112012 (2014) doi:10.1103/PhysRevD.90.112012 [arXiv:1410.2589 [astro-ph.HE]].

[42] T. Arlen et al. [VERITAS Collaboration], Astrophys. J. 757, 123 (2012) doi:10.1088/0004-637X/757/2/123 [arXiv:1208.0676 [astro-ph.HE]].

[43] S. Desai et al. [Super-Kamiokande Collaboration], Phys. Rev. D 70, 083523 (2004) [Phys. Rev. D 70, 109901 (2004)] doi:10.1103/PhysRevD.70.083523, 10.1103/PhysRevD.70.109901 [hep-ex/0404025].

[44] M. G. Aartsen et al. [IceCube Collaboration], Phys. Rev. Lett. 110, no. 13, 131302 (2013) doi:10.1103/PhysRevLett.110.131302 [arXiv:1212.4097 [astro-ph.HE]].

[45] O. Adriani et al. [PAMELA Collaboration], Nature 458, 607 (2009) doi:10.1038/nature07942 [arXiv:0810.4995 [astro-ph]].

[46] M. Aguilar et al. [AMS Collaboration], Phys. Rev. Lett. 110, 141102 (2013). doi:10.1103/PhysRevLett.110.141102 
[47] O. Adriani et al. [PAMELA Collaboration], Phys. Rev. Lett. 105, 121101 (2010) doi:10.1103/PhysRevLett.105.121101 [arXiv:1007.0821 [astro-ph.HE]].

[48] N. Arkani-Hamed, D. P. Finkbeiner, T. R. Slatyer and N. Weiner, Phys. Rev. D 79, 015014 (2009) doi:10.1103/PhysRevD.79.015014 [arXiv:0810.0713 [hep-ph]].

[49] D. Hooper and T. M. P. Tait, Phys. Rev. D 80, 055028 (2009) doi:10.1103/PhysRevD.80.055028 [arXiv:0906.0362 [hep-ph]].

[50] D. Hooper and L. Goodenough, Phys. Lett. B 697, 412 (2011) doi:10.1016/j.physletb.2011.02.029 [arXiv:1010.2752 [hep-ph]].

[51] K. N. Abazajian and M. Kaplinghat, Phys. Rev. D 86, 083511 (2012) [Phys. Rev. D 87, 129902 (2013)] doi:10.1103/PhysRevD.86.083511, 10.1103/PhysRevD.87.129902 [arXiv:1207.6047 [astro-ph.HE]].

[52] C. Gordon and O. Macias, Phys. Rev. D 88, no. 8, 083521 (2013) [Phys. Rev. D 89, no. 4, 049901 (2014)] doi:10.1103/PhysRevD.88.083521, 10.1103/PhysRevD.89.049901 [arXiv:1306.5725 [astro-ph.HE]].

[53] T. Daylan, D. P. Finkbeiner, D. Hooper, T. Linden, S. K. N. Portillo, N. L. Rodd and T. R. Slatyer, arXiv:1402.6703 [astro-ph.HE].

[54] F. Calore, I. Cholis and C. Weniger, JCAP 1503, 038 (2015) doi:10.1088/1475-7516/2015/03/038 [arXiv:1409.0042 [astro-ph.CO]].

[55] For more detail, see: Simona Murgia, these proceedings (2015).

[56] For more detail, see: Troy Porter, these proceedings (2015).

[57] M. Ajello et al. [Fermi-LAT Collaboration], arXiv:1511.02938 [astro-ph.HE].

[58] P. Agrawal, B. Batell, P. J. Fox and R. Harnik, JCAP 1505, 011 (2015) doi:10.1088/1475-7516/2015/05/011 [arXiv:1411.2592 [hep-ph]].

[59] C. Boehm, M. J. Dolan, C. McCabe, M. Spannowsky and C. J. Wallace, JCAP 1405, 009 (2014) doi:10.1088/1475-7516/2014/05/009 [arXiv:1401.6458 [hep-ph]].

[60] M. Abdullah, A. DiFranzo, A. Rajaraman, T. M. P. Tait, P. Tanedo and A. M. Wijangco, Phys. Rev. D 90, 035004 (2014) doi:10.1103/PhysRevD.90.035004 [arXiv:1404.6528 [hep-ph]].

[61] A. Martin, J. Shelton and J. Unwin, Phys. Rev. D 90, no. 10, 103513 (2014) doi:10.1103/PhysRevD.90.103513 [arXiv:1405.0272 [hep-ph]].

[62] D. Bauer et al. [Snowmass 2013 Cosmic Frontier Working Groups $1 Đ 4$ Collaboration], Phys. Dark Univ. 7-8, 16 doi:10.1016/j.dark.2015.04.001 [arXiv:1305.1605 [hep-ph]].

[63] S. Arrenberg et al., arXiv:1310.8621 [hep-ph].

[64] M. Cahill-Rowley, R. Cotta, A. Drlica-Wagner, S. Funk, J. Hewett, A. Ismail, T. Rizzo and M. Wood, Phys. Rev. D 91, no. 5, 055011 (2015) doi:10.1103/PhysRevD.91.055011 [arXiv:1405.6716 [hep-ph]].

[65] D. N. Spergel and P. J. Steinhardt, Phys. Rev. Lett. 84, 3760 (2000) doi:10.1103/PhysRevLett.84.3760 [astro-ph/9909386].

[66] M. Boylan-Kolchin, J. S. Bullock and M. Kaplinghat, Mon. Not. Roy. Astron. Soc. 415, L40 (2011) [arXiv:1103.0007 [astro-ph.CO]].

[67] For recent discussion, see: M. Kaplinghat, S. Tulin and H. B. Yu, arXiv:1508.03339 [astro-ph.CO]. 
[68] J. M. Cline, Z. Liu, G. Moore and W. Xue, Phys. Rev. D 90, no. 1, 015023 (2014) doi:10.1103/PhysRevD.90.015023 [arXiv:1312.3325 [hep-ph]].

[69] K. K. Boddy, J. L. Feng, M. Kaplinghat and T. M. P. Tait, Phys. Rev. D 89, no. 11, 115017 (2014) doi:10.1103/PhysRevD.89.115017 [arXiv:1402.3629 [hep-ph]].

[70] K. K. Boddy, J. L. Feng, M. Kaplinghat, Y. Shadmi and T. M. P. Tait, Phys. Rev. D 90, no. 9, 095016 (2014) doi:10.1103/PhysRevD.90.095016 [arXiv:1408.6532 [hep-ph]].

[71] Y. Hochberg, E. Kuflik, H. Murayama, T. Volansky and J. G. Wacker, Phys. Rev. Lett. 115, no. 2, 021301 (2015) doi:10.1103/PhysRevLett.115.021301 [arXiv:1411.3727 [hep-ph]]. 